\title{
An Approach for Crack Detection in Beams on Elastic Foundations using Frequency Measurements
}

\author{
D.P. Patil and S.K. Maiti \\ Department of Mechanical Engineering, Indian Institute of Technology Bombay, Mumbai, 400 076, India
}

(Received 17 December 2001; revised 12 July 2002; accepted 16 July 2002)

\begin{abstract}
In this paper a method has been proposed for the detection of open cracks based on frequency measurements on slender beams' on continuous and discontinuous elastic foundations. The cracks are represented by massless rotational springs. The foundation is represented by a Winkler spring. The approach exploits the transfer matrix method (TMM) to advantage and assumes slender beams in which the shear deformation and rotational inertia are negligible. Both forward and inverse problems are solved and results are presented. In forward problems, the frequencies are determined by giving the rotational spring stiffness as an input. In the inverse analysis, a method is employed to detect the location and size of a crack by providing the natural frequencies as inputs. Numerical case studies are presented to demonstrate the method's effectiveness. The natural frequencies required for the inverse analysis are computed using a finite element program. A three-node interface finite element is used to take care of the foundation stiffness. It appears finally that the method can detect the location of a normal crack of size $10 \%$ or more of the section depth with an error less than $3 \%$. The crack size can also be predicted with an error less than about $6 \%$.
\end{abstract}

\section{INTRODUCTION}

The vibration - based detection of the location and size of cracks in slender beams has been mostly applied so far to single span beams, which can be uniform in section or geometrically segmented, and have internal or external cracks, etc. ${ }^{1-9}$ The choice of using the natural frequency as a basis in the development of NDE is most attractive. This is due to the fact that the natural frequencies of a beam can be measured from one single location in the beam, thus offering scope for the development of a fast and global NDE technique. There are now considerable efforts being made in this direction to make the method useful in practice. The method of representing an open crack by a massless rotational spring is found to be very effective for a crack size of more than $10 \%$ of its section depth..$^{2-9}$ This range of effectiveness can be exploited gainfully in the case of redundant structures. ${ }^{2}$ There are many applications, e.g. rails of railway tracks, pipelines anchored at regular/irregular intervals, etc., where the supports have flexibility. It is worthwhile examining how the method of crack detection can be extended to beams of this type. Though the problem is important, only a few investigations have been published on this issue. ${ }^{6,7}$ These have provided the main motivation for the present study.

Liang et al. ${ }^{6}$ have given a trial and error method for the prediction of the location of cracks in a beam on an elastic foundation using the natural frequencies. The cracks in the beam are evaluated through the change in stiffness of the beam segment. Hasan ${ }^{7}$ proposes a perturbation technique for calculating the changes in natural frequency due to a small crack $(a / h \leq 0.1 ; a$ is the crack size and $h$ is the section depth). Thereby only the forward problem is addressed. The forward problem involves the determination of the natural frequencies given the geometric details. The inverse problem concerns the determination of the location and/or size of the crack from a knowledge of the natural frequencies.
It has been shown recently that the transfer matrix method is applicable to beams with a large number of geometric segments. ${ }^{8}$ This method offers definite advantages over the traditional method, i.e. the normal mode approach ${ }^{3,5}$ or FEM. $^{9}$ The characteristic determinant is of size $(2+n) \times(2+n)$, where $n$ is the number of intermediate supports. It helps to reduce the requirement of computational storage and time. How this method can be exploited in handling such beam configurations to examine the inverse problem is open for investigation. The present study is motivated by such an issue. The forward problem is examined in passing.

The amount of effort that is involved in predicting the location and size ( $a>10 \%$ of section depth) of a crack based on the open crack model is much less than that involved using the 'closed' or 'breathing' crack model. ${ }^{10-11}$ The open crack model, in contrast to the breathing or closing crack model, is reported to give rise to an underprediction of crack size. ${ }^{11}$ The analysis based on the open crack model can be used to give a quick and initial estimate of location and size. The breathing crack analysis, or any of the standard conventional NDT techniques, can be used, if necessary, for an improvement in the initial predictions. Their use lead to a considerable saving in time, labour and cost for long beam - like components, such as rails, pipelines, etc.

In the present study the transfer matrix method is coupled with the rotational spring model to solve both forward and inverse problems of beams on elastic foundation with open crack located normally to its axes. The beams are considered to be slender so that shear deformation and rotational inertia can be neglected. Damping in the beam is neglected. The method of modelling is restricted to open normal edge cracks. The foundation stiffness is represented by a Winkler spring. Numerical studies are presented to demonstrate the accuracy in both the cases. The natural frequencies required for solving the inverse problem are computed using a finite element program. ${ }^{13}$ 